\title{
Segmentation of Neuronal-Cell Images from Stained Fields and Monomodal Histograms
}

\author{
Tuan D. Pham ${ }^{1}$ and Denis I. Crane ${ }^{2}$ \\ ${ }^{1}$ School of Information Technology \\ James Cook University \\ Townsville, QLD 4811, Australia \\ ${ }^{2}$ School of Biomolecular and Biomedical Science \\ Griffith University \\ Nathan, QLD 4111, Australia
}

\begin{abstract}
Information from images taken of cells being grown in culture with oxidative agents allows life science researchers to compare changes in neurons from the Zellweger mice to those from normal mice. Image segmentation is the major and first step for the study of these different types of processes in cells. In this paper we develop an innovative strategy for the segmentation of neuronal-cell images which are subjected to stains and whose histograms are monomodal. Such nontrival images make it a challenging task for many existing image segmentation methods. We show that the proposed method is an effective and simple procedure for the subsequent quantitative analysis of neuronal images.
\end{abstract}

\section{INTRODUCTION}

Neurodegeneration refers to the excessive damage or loss of neurons, or brain and spinal cord cells which perform different functions such as controlling movement, processing sensory information, and making decisions. Neurodegenerative diseases can cause devastating effects on an individual. We are particularly interested in using image analysis to help our study of the neurodegeneration in a human disorder called Zellweger syndrome.

In our study, the cells are from mice that are a model of the Zellweger syndrome, a severe neurodegenerative disorder characterised by death in the first 1-6 months after birth, severe dysmorphia, hypotonia, and other widespread tissue defects. This disorder arises because of defects in cellular organelles called peroxisomes, that are required for a number of essential cellular metabolic functions. We have hypothesised that the loss of peroxisomes in neurons results in these cells being susceptible to oxidative stress, because peroxisomes contain a number of important anti-oxidant enzymes, including catalase needed to break down hydrogen peroxide that is made in cells. In response to oxidative stress, we propose that these neurons will deteriorate. In morphological terms, we expect to see this initial deterioration as the contraction, and eventually loss, of processes of neurons grown in culture.

Given the above motivation, image analysis by segmentation is an efficient way that allows us to measure the changes in cell process number and length from images taken of cells being challenged in culture with oxidative agents. By utilizing techniques for image processing, we will be able to precisely compare changes in neurons from the Zellweger mice to those from normal mice in a quantitative manner.

Methods for image segmentation can be classified into four main categories: edge detection, thresholding, region-based segmentation, and morphological transform [1]. Application of each approach can be useful for solving some particular problem. However, in general, segmentation of nontrival images is still one of the most difficult task in image processing.

Algorithms for removing shading fields from images define an image as the product of a reflectance function $r(x, y)$ (intrinsic image) and an illunination function $i(x, y)$ :

$$
f(x, y)=r(x, y) i(x, y)
$$

Taking the logarithm of (1) we have

$$
\hat{f}(x, y)=\hat{r}(x, y)+\hat{i}(x, y)
$$

where $\hat{f}(x, y)=\ln f(x, y), \hat{r}(x, y)=\ln r(x, y)$, and $\hat{i}(x, y)=\ln i(x, y)$.

If $\hat{f}(x, y), \hat{r}(x, y)$ and $\hat{i}(x, y)$ are thought of as random variables and $u=\hat{r}+\hat{i}$, then under the law of probability we can obtain the following probability density function (pdf) [2]

$$
p_{\hat{f}}(u)=\int_{-\infty}^{+\infty} p_{\hat{r}}(u-\hat{i}) p_{\hat{i}}(\hat{i}) d \hat{i}
$$

which shows that the pdf of $\hat{f}$ is equal to the convolution of the two pdfs of $\hat{i}$ and $\hat{r}$.

If the illumination is uniform, then both $i(x, y)$ and $\hat{i}(x, y)$ are constant, we obtain $p_{\hat{f}}(u)=p_{\hat{r}}(u)$. However, if the illumination is not uniform then the histogram (pdf) is distorted and conventional thresholding methods cannot be directly applied.

There are some advanced methods being recently developed for extracting image information from shading fields or under variable illumination. Weiss [3] proposed an algorithm 


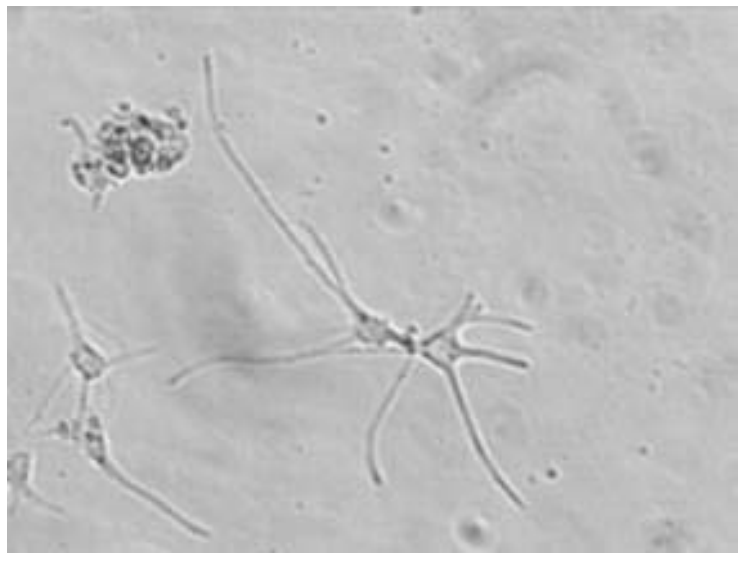

Fig. 1. Nerve cells taken at 15 minutes after culture

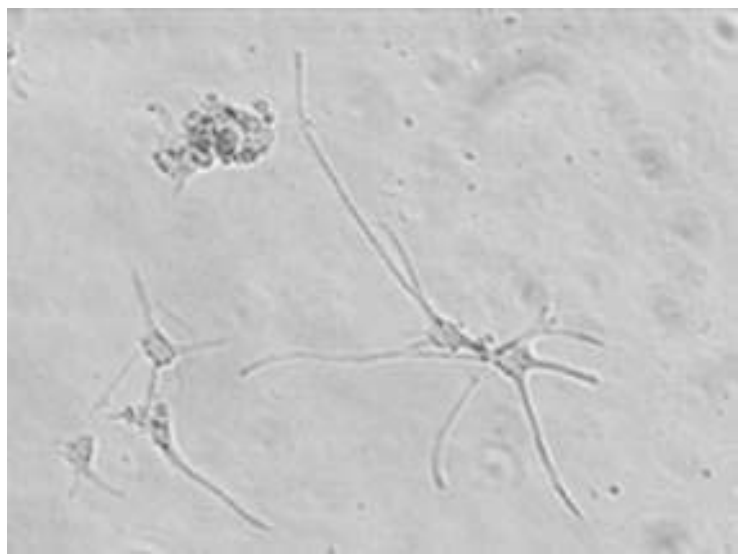

Fig. 2. Nerve cells taken at 30 minutes after culture

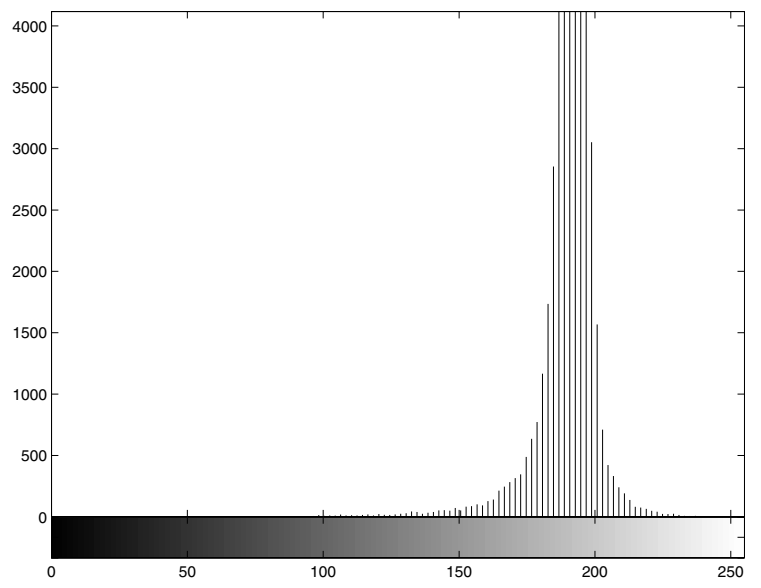

Fig. 3. Histogram of image shown in Figure 1

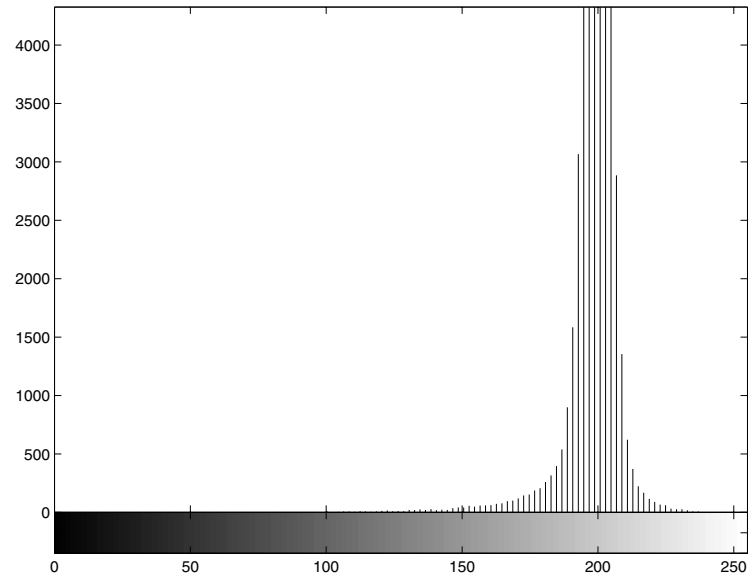

Fig. 4. Histogram of image shown in Figure 2

for extracting intrinsic images. This method requires a sequence of static scene images in different lighting conditions. Although this method can be effective for deriving intrinsic images from shading, it is not effective for removing vague shadows. Finlayson et al. [4] developed a method for removing shadows from colour images. This method can extract the intrinsic image from a single RGB image, but it also requires that the shadow fields must have distinguishing boundaries.

In the present study, the neuronal-cell images consist of not only vague stained fields but also monomodal histograms which make both edge detection and thresholding algorithms break down. In the following sections, we will present a novel approach for the segmentation of these images and show that it is effective for handling such difficult task.

\section{Proposed Methodology}

Consider two images as shown in Figures 1 and 2 whose histograms are shown in Figures 3 and 4 respectively, in which the neurons (nerve cells) are the objects to be extracted from the images. Due to strong similarity between the object and background pixel values, both image histograms appear to be monomodal. In addition, the stained fields are also similar to the objects. We applied different edge detection methods including Sobel, Canny, Prewitt, Robert, zero-crossing, and the Laplacian of Gaussian filter [1], [5] to the images of Figures 1 and 2. We found the Sobel edge detection provided the best results. We also applied several techniques for the segmentation of Figures 1 and 2. These techniques include minimum error thresholding [2], fuzzy $c$-means algorithm [6], morphological watershed [1], and Otsu's method [7]. We found that Otsu's thresholding method, which is based on discriminant analysis to maximize the separability of the two populations, produced the best segmentation results so far. We note that the vague boundaries of the stained fields may represent a third class for the implementation of the fuzzy $c$-means algorithm in which $c=3$ as we studied the segmentation of cell puncta [8]. However, we found that the results are more spurious than those obtained from Otsu's method. 
In general, we have observed that thresholding methods tend to misclassify stained fields as object pixels; whereas edge detection operators miss out the object boundaries which are in the proximity of the stained fields. Thus, the idea is that if we can identify the pixel values of the stained fields then we can better carry out the segmentation of these images by using the mean value of the stained fields as a threshold for binary classification. We discuss our proposed strategy which is very simple and effective for finding this threshold by making use of the Sobel edge operator and Otsu's method as follows.

Let a binary image which is obtained by using Otsu's thresholding method be denoted as $f_{O}(x, y)$ whose elements are the object pixels; and the binary Sobel edge image as $f_{S}(x, y)$ whose elements are the edge pixels of the object.

We apply a mask, denoted by $M_{S}$, of size $h_{x} \times h_{y}$ over image $f_{S}(x, y)$, where $(x, y)$ is the middle pixel coordinate pair of $M_{S}$. If we choose the mask size of $3 \times 3$, then $M_{S}=\left[f_{S}(x-1, y-1), f_{S}(x-1, y), f_{S}(x-\right.$ $1, y+1) ; f_{S}(x, y-1), f_{S}(x, y), f_{S}(x, y+1) ; f_{S}(x+1, y-$ $\left.1), f_{S}(x+1, y), f_{S}(x+1, y+1)\right]$.

We wish to identify the set $Q=\left\{f_{\omega}(x, y)\right\} \in \Omega$, where $\Omega$ is the space of stained pixels.

We can determine the elements of $Q$ by classifying

$$
f_{O}(x, y) \text { as } f_{\omega}(x, y)
$$

if

$$
f_{O}(x, y) \cup M_{S}=\emptyset
$$

The idea can be explained in that the segmented image $f_{O}(x, y)$ has turned off most of the background pixels in $f(x, y)$ and consists of narrow object regions which are the shapes of the neurites. Therefore the idea of applying the mask $M_{S}$ over the edge image $f_{S}(x, y)$ and then comparing $M_{S}$ with the segmented image $f_{O}(x, y)$ is to search for the stained pixels which are misclassified by the thresholding method and in the proximity of the edges. The search domain depends on the size of $M_{S}$.

After obtaining a set of the stained pixels, $Q=\left\{f_{\omega}(x, y)\right\}$, we select a new threshold, denoted by $T$, by taking the average value of the identified stained pixels:

$$
T=\frac{1}{|Q|} \sum_{(x, y) \in Q} f_{\omega}(x, y)
$$

where $|Q|$ is the cardinality of $Q$.

We describe the procedure for finding threshold $T$ as follow.

\section{Algorithm}

1) Given image $f(x, y)$ of size $M \times N$, and mask $M_{S}$ of size $h_{x} \times h_{y}$

2) Obtain edge image $f_{S}(x, y)$ by Sobel edge operator

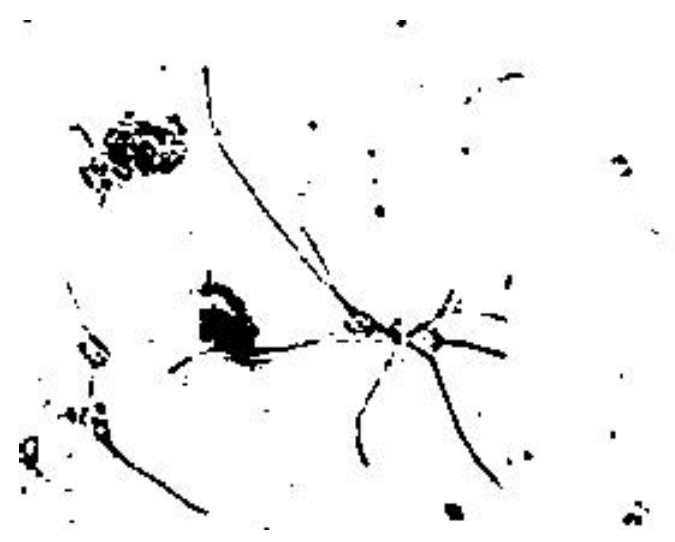

Fig. 5. Segmentation of image shown in Figure 1 by Otsu's method

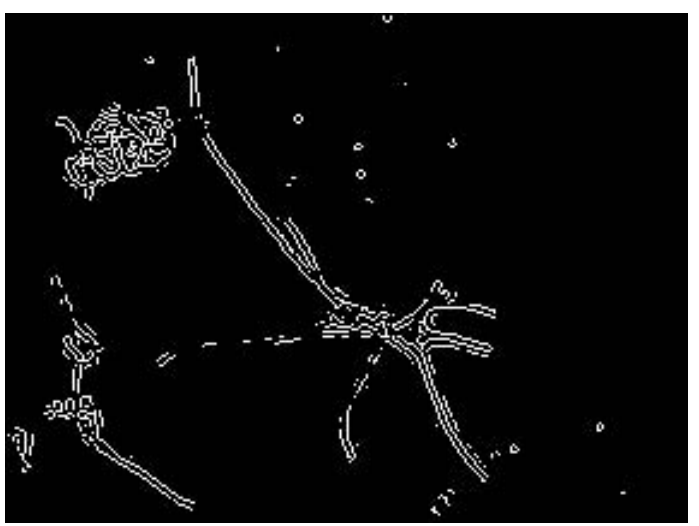

Fig. 6. Sobel edge of image shown in Figure 1

3) Obtain initial segmented image $f_{O}(x, y)$ by Otsu's thresholding method

4) Start loops $x=1$ to $M$; $y=1$ to $N$ :

5) Apply $M_{S}$ over $f_{S}(x, y)$ to check if $M_{S}$ contains any edge pixels at $(x, y)$

6) Assign $f_{O}(x, y)$ as $f_{\omega}(x, y)$ if no edge is found in $M_{S}$ at $(x, y)$

7) End loops

8) Compute new threshold $T$ using (4)

9) Segment $f(x, y)$ using $T$.

\section{RESULTS}

The images shown in Figures 1 and 2 are of neuronal cells that have been grown in culture from mouse brain tissue cells, and taken at 15 minutes and 30 minutes after the culture respectively. These cells will typically have a central cell body and a number of processes extending from the cell body.

We applied the proposed algorithm to binarize the two images by assigning each pixel in each image to either one of the two classes (object and background) based on the threshold $T$ given in (4). In both experiments, we used a $3 \times 3$ mask to search for the edge pixels. Figures 5-7 show the Otsu segmented image, Sobel edge image, and final segmented image of Figure 1 respectively. Due to the monomodel 


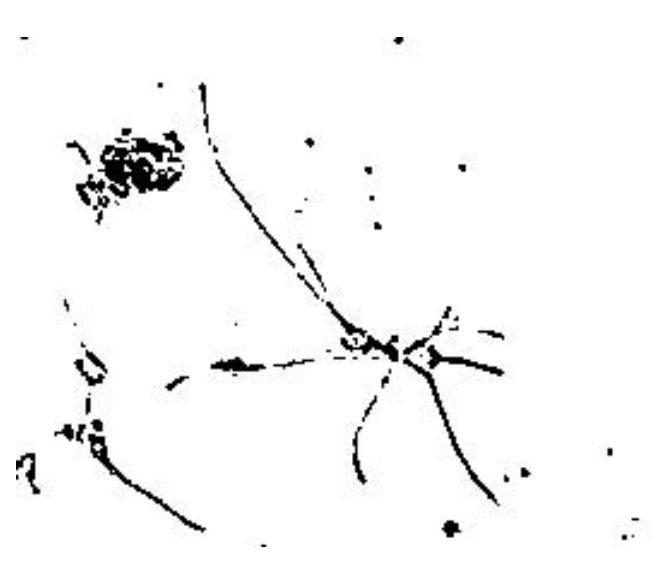

Fig. 7. Final segmentation of image shown in Figure 1

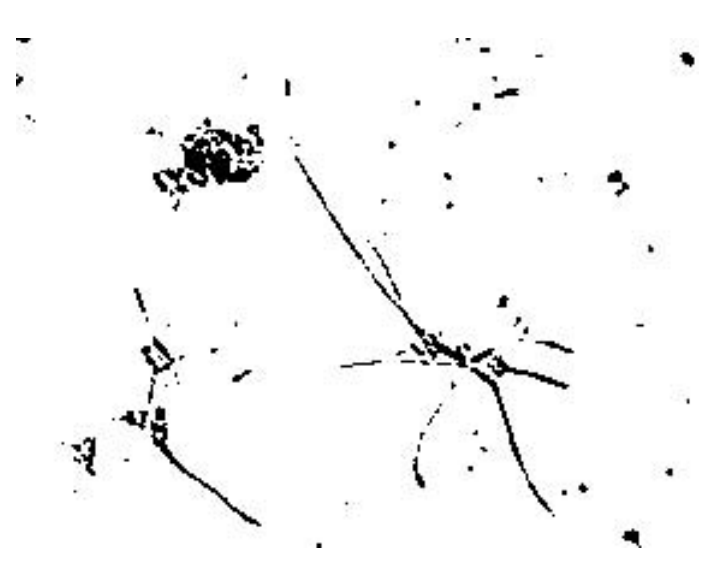

Fig. 8. Segmentation of image shown in Figure 2 by Otsu's method

histogram of Figure 1, the segmentation by Otsu's method misclassified many stained pixels as object pixels; whereas the Sobel edge method failed to detect true edges about the overlap of stained and neurite pixels. Our proposed strategy could remove the stained fields contained by Otsu's segmentation and recover some neurite edges missed out by the Sobel edge operator.

Figures 8-10 show the segmented image by Otsu's method, Sobel edge image, and final segmented image of Figure 2 respectively. For this image, it can be observed that the pixel levels of the stained fields are between those of the object pixels. The threshold level obtained from Otsu's method has a bias toward darker pixel values, and therefore missed out brighter object pixels. Similarly, the Sobel edge detector could not recognize the boundary of the neuronal pixels whose values are relatively brighter than the majority. Our proposed procedure could identify a set of stained pixels and used their average value as a new threshold for segmentation. As a result, Figure 10 shows that some neuronal segments were recovered but some stained spots highlighted. However, after using some method for edge linking [1], these spots can be easily identified and further removed by comparing the sizes of the neuronal regions and isolated stained fields.

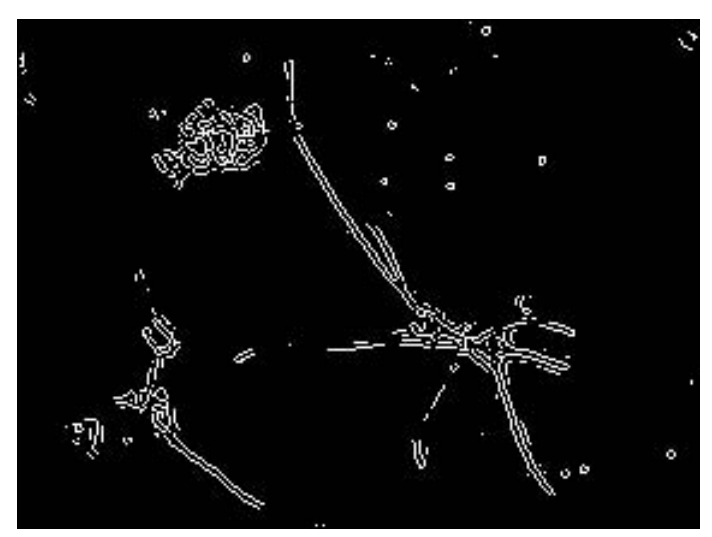

Fig. 9. Sobel edge of image shown in Figure 2

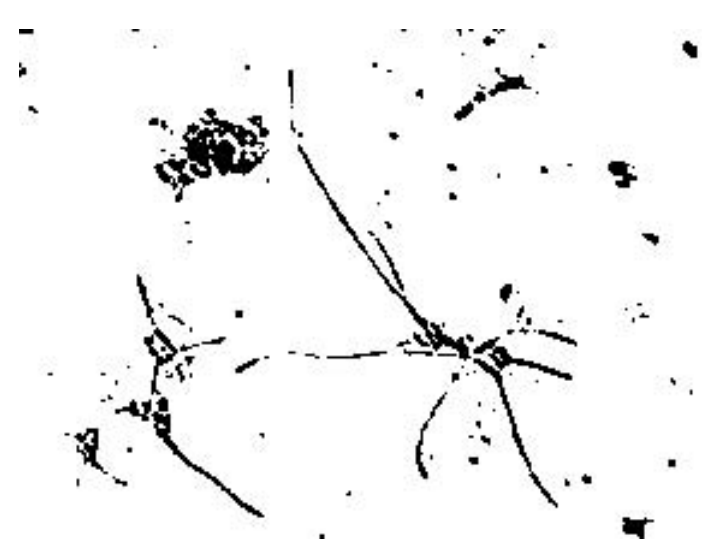

Fig. 10. Final segmentation of image shown in Figure 2

\section{Conclusion}

The emerging technology of molecular imaging provides innovative approaches for medicine and biology research [9]. We have presented an effective and simple procedure for extracting the neuronal information from images having vague stained fields and monomodal histograms which make it a very difficult task for either individual thresholding, edge detection, or intrinsic image deriving methods.

\section{REFERENCES}

[1] R.C. Gonzalez, and R.E. Woods, Digital Image Processing, 2nd edition. New Jersey: Prentice Hall, 2002.

[2] M. Petrou, and P. Bosdogianni, Image Processing: The Fundamentals. New York: Wiley, 2000.

[3] Y. Weiss, Deriving intrinsic images from image sequences, Proc. Int. Conf. Computer Vision, pp. 68-75, 2001.

[4] G.D. Finlayson, S.D. Hordley, and M.S. Drew, Removing shadows from images, Proc. European Conf. Computer Vision, vol. 4, pp. 823836, 2002.

[5] J.S. Lim, Two-Dimensional Signal and Image Processing. New Jersey: Prentice Hall, 1990.

[6] J.C. Bezdek, Pattern Recognition with Fuzzy Objective Function Algorithms. New York: Plenum Press, 1981.

[7] N. Otsu, A thresholding selection method from gray-level histograms, IEEE Trans. Systems, Man, and Cybernetics, vol. 9, pp. 62-66, 1979.

[8] T.D. Pham, D. Crane, T.H. Tran, and T.H. Nguyen, Extraction of fluorescent cell puncta by adaptive fuzzy segmentation, Bioinformatics, vol. 20, pp. 2189-2196, 2004.

[9] Special Issue on: Molecular Imaging: Emerging Technology \& Biomedical Applications, Proceedings of the IEEE, vol. 93:4, 2005. 